\title{
IWF Based Optimization of Porous Insert Configurations for Heat Transfer Enhancement using CFD
}

\author{
T. Sathish ${ }^{1 \dagger}$ and V. Mohanavel $^{2}$ \\ ${ }^{1}$ Vesta Research Institute, Aranthangi, Tamil Nadu, India \\ ${ }^{2}$ Department of Mechanical Engineering, Kingston Engineering College, Vellore, Tamil Nadu, India \\ †Corresponding Author Email: sathish.sailer@gmail.com
}

(Received May 15, 2018; accepted July 18, 2018)

\begin{abstract}
In this paper, a new optimization method coupling genetic algorithm (GA) and computational fluid dynamics (CFD) based on Windows Socket was found to optimize the configurations of porous insert in a tube for heat transfer enhancement. The region in the enhanced tube was divided into several layers in the radial direction. The porosity of porous media filled in each layer was the design variable, which varies from 0.5 to 1.0 . The results show that the thermo-hydraulic performance of the enhanced tube can be improved effectively by using the optimized porous insert, particularly using the optimized multiple layers of porous insert. However, there is an appropriate layer number of porous insert to ensure the optimal performance of the enhanced tube for a given set of parameters.
\end{abstract}

Keywords: Heat transfer enhancement; Porous insert; Genetic algorithm (GA); Computational fluid dynamics (CFD); Optimization.

\section{INTRODUCTION}

Porous media are used in a wide variety of natural and manmade systems including biological systems, electronic cooling, heat exchangers, thermal storages, solar collectors and thermal insulation (Mahmoudi and Karimi 2014, Sathish 2017a). Deep understanding of the flow and heat transfer mechanisms in porous media is one of the main prerequisites for the effective use of porous media (Vafai, 2009; Sathish, 2017b).

Until now, a lot of researches about the flow and heat transfer in porous media have focused on the layout of porous media to improve the thermal or thermo-hydraulic performance. For example, Morosu (2005) numerically analyzed the thermodynamic performance of a tube completely or partially filled with porous media.

The porous layer was attached on the inner surface of the tube or inserted at the core of the tube. Their results showed that the maximum entropy generation occurs at the interface between the clear flow and flow in porous media. Bhargavi and Satyamurty (2011) analytically investigated the enhancement of forced convective heat transfer for laminar flow in a parallel plate channel partially filled with porous media (Sathish and Jayaprakash, 2017b).
The porous layer was attached to one or two of the walls or inserted at the core of the channel. Their results showed that inserting the porous layer at the core had maximum enhancement in heat transfer per unit pressure gradient. Yang et al. (2011) studied the heat transfer enhancement for the flow in a pipe partially filled with porous media, and found that the velocity of flow affected the optimal filling position. However, most of the above studies only focused on the parameter analysis, and are not really optimization researches (Sathish, and Jayaprakash, 2015).

On the other hand, for the flow and heat transfer in a clear tube or channel, Guo and his coworkers (Tao, W.Q., et al. 2002; Zheng, Z.Z., et al. 2014; He, Y.L., and Tao, W.Q., 2014) proposed the field synergy principle and the entransy theory, and deduced the optimization equations based on the variational method. Similarly, Bejan (2004) deduced the optimization equations based on the constructal theory, and introduced the constructal theory into the optimization of flow and heat transfer in porous media (Sathish and Jayaprakash 2017a). However, sometimes it is difficult to deduce the optimization equations for some complicated flow and heat transfer problems. The optimizing method based on various optimization algorithms is a good alternative solution ( $\mathrm{Xi}, 2013$, Sathish 
and Muthulakshmanan 2018). Godwin (2017) and Lakshmanan (2017) investigated about the optimum parameters for obtaining the best performance using alternate fuels of IC engines working under the current cooling system using Nanofluids. Srinivasan (2017) studied and analyzed about the missile with grid fins and the effect on flow drag using ANSYS. Venkatesh et al. (2016) studied the flow of heat and fluid in solar air heater using Computational Fluid Dynamics (CFD). The predicted temperature results were observed within a deviation of $\pm 10.64 \%$.

In this paper, the heat transfer enhancement for convection heat transfer of laminar flow in a circular tube partially or completely filled with porous media was numerically investigated. A new optimization method combining IWF and CFD was proposed to optimize the configurations of porous insert to get a better thermo-hydraulic performance. In the procedure of optimization, the programs of IWF and CFD ran independently and were coupled by the Windows Socket. Therefore, this optimization method can be easily extended to other flow and heat transfer problems and has an excellent prospect.

\section{PHYSICAL MODEL}

A two-dimensional axisymmetric numerical model for convection heat transfer of steady laminar flow in a circular tube with constant heat flux is considered. As shown in Fig. 1, the half of region in the enhanced tube is divided into Nlayers in the radial direction. Each layer is filled with porous media with different porosity. The radius $\left(\mathrm{r}_{0}\right)$ and length $(\mathrm{L})$ of the enhanced tube are $0.01 \mathrm{~m}$ and 0.4 $\mathrm{m}$, respectively.

An auxiliary heating segment with a length of $0.6 \mathrm{~m}$ is placed before the inlet of the enhanced tube to make sure that the fluid is fully developed for flow and heat transfer in the enhanced tube. To simplify the analysis, some assumptions in the simulation are listed as follows:

(1) the porous media is regarded as homogeneous and isotropic,

(2) the gas phase and the solid phase in porous media exist in a state of local thermal equilibrium;

(3) the Forchheimer-Brinkman model is used to describe the flow in the porous region.

\section{MATHEMATICAL MODEL}

\subsection{Governing Equations}

The governing equations including the continuity, momentum, and energy can be expressed as follows (Maerefat, M., et al. 2010);

Continuity equation:

$\frac{\partial(\rho u)}{\partial x}+\frac{1}{r} \frac{\partial(r \rho v)}{\partial r}=0$

\subsection{Boundary Conditions}

As shown in Fig. 1, the boundary conditions can be expressed as follows:

(1) inlet boundary: constant velocity and temperature condition.

$u=u_{\text {in }}, v=0, T=T_{\text {in }}=293 \mathrm{~K}$

(2) outlet boundary: fully-developed condition.

$\frac{\partial u}{\partial x}=\frac{\partial v}{\partial x}=\frac{\partial T}{\partial x}=0$

(3) upper boundary: constant heat flux condition. $\mathrm{u}=\mathrm{v}=0, \mathrm{q}=\mathrm{kw} @ \mathrm{~T} / @ \mathrm{r}=100 \mathrm{Wm} 2$

$u=v=0, q=\lambda_{w} \frac{\partial T}{\partial r}=100 \mathrm{Wm}^{-2}$

(4) lower boundary: axisymmetric condition @u/@r=@v/@r=@T/@r=0.

$\frac{\partial u}{\partial r}=\frac{\partial v}{\partial r}=\frac{\partial T}{\partial r}=0$

\subsection{Numerical Methods}

The pre-processing software GAMBIT 2.2.3 is used to create the geometric model and generate mesh. A uniform grid solution is used to eliminate the impact of grid partitioning method on the simulation results. The porous insert configuration is controlled by setting the parameters of each cell.

The finite volume method is used to solve the governing equations (He, Y.L., et al. 2011), all equations are numerically solved using the commercial CFD software FLUENT 6.3 (Fluent Incorporated 2003). The second upwind scheme is used to discretize the convective terms in momentum and energy equations, and the SIMPLE algorithm (Tao, W.Q 2001) is used to ensure the coupling between velocity and pressure. The coupled set of governing equations is solved iteratively with the convergence criterion set to 106. Additionally, the thermophysical properties of air under the temperature of $293.15 \mathrm{~K}$ are selected (Sivakumar, V., et al. 2017).

In order to analyze the thermal-hydraulic characteristics of the enhanced tube, some parameters are defined as follows:

The Reynolds number, Nusselt number and friction factor are given as:

$$
\begin{aligned}
& \mathrm{Re}=\frac{2 \rho u r_{0}}{\mu}, \\
& N u=\frac{2 h r_{0}}{\lambda_{f}}, \\
& f=\frac{\Delta p}{L} \frac{2 r_{0}}{(1 / 2) \rho u^{2}}
\end{aligned}
$$

where, $\mathrm{h}$ is the average heat transfer coefficient, $\Delta p$ is the differential pressure between inlet and outlet.

The performance evaluation criterion (PEC) of heat 
transfer enhancement under constant pumping power is defined as (Cheng Z.D., et al. 2012):

$P E C=\frac{N u / N u_{c}}{\left(f / f_{c}\right)^{1 / 3}}$

where, the subscript “c"' represents clear tube without porous media.

\subsection{Code Checking and Models Validation}

To ensure that the calculation results are reliable, the grid independency test and model validation are carried out. The thermal-hydraulic characteristics for fully developed laminar flow in the enhanced tube shown in Fig. 1 without filled porous media are investigated, since the uniform grid solution is used for clear tube and the enhanced tube with different porous inserts.

Five different grid systems are tested namely 20 (radial) $\times 400$ (axial), $25 \times 500,33 \times 666,50 \times$ $1000,100 \times 2000$. For the fully developed laminar flow in a pipe with constant heat flux boundary condition, the theory analysis values for $\mathrm{Nu}$ and $\mathrm{f} \bullet \mathrm{Re}$ are 4.36 and 64, respectively (Yang, S.M., and Tao, W.Q., 2006). The difference of $\mathrm{Nu}$ and $\mathrm{f} \bullet$ Re between the fifth grid system and the fourth grid system are less than $0.03 \%$ and $0.05 \%$, respectively.

Therefore, the fifth grid system is chosen in making a trade-off between the accuracy and the CPU time. The difference of $\mathrm{Nu}$ and $\mathrm{f} \bullet \mathrm{Re}$ between the fifth grid system and the analytical value are less than $0.05 \%$ and $0.00156 \%$, which proves the validity of the flow and heat transfer numerical model of the enhanced tube.

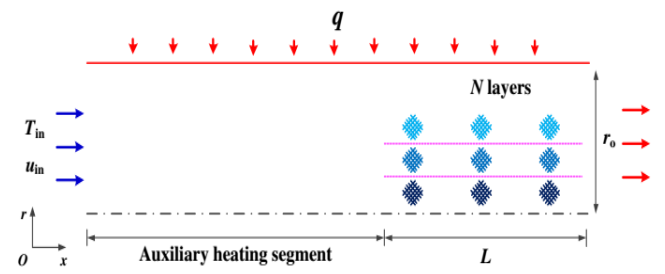

Fig. 1. Schematic diagram of the present problem.

\section{Optimization Procedure}

The process of how the configurations of porous insert in the enhanced tube are optimized by IWF, and how the IWF is coupled with CFD are presented as follow.

As shown in Fig. 1, the half of region in the enhanced tube is divided into $\mathrm{N}$ layers in the radial direction. The porosity for each layer is the design variable, which varies from 0.5 to 1.0 . Therefore, there are $\mathrm{N}$ design variables for the optimization problem in this paper.

\subsection{Inertia Weight Firefly Algorithm}

The critical objective is to minimizs the greatest of support value and minimize the base of support value. For that, we have utilized some control parameters such as Initial brightness $b=0.4$; Randomization parameter $\eta=0.5$ Coefficient of light absorption $\gamma=0.5$.

A multi-objective function is mathematically formulated as,

$\operatorname{obj}(i)=\min \{x\}$

In the above equation (4), $X$ denoted data rate value.

Calculation of the fitness function:

$F(j)=\left\{\frac{1}{(1+o b j(i))} ;\right.$ if $(\operatorname{obj}(i)) \geq 0$

$\operatorname{obj}(i)$ - Separate objective functions

Numbers of iterations are used for calculating the Fitness function. This iteration will be continuing until the best-optimized value is as received. The objective function should be high in our work.

As attractiveness of fireflies' is directly proportional to the light intensity seen by adjacent firefly, we can now define the attractiveness $\beta$ a firefly by

$\beta(r)=\beta_{0} e^{-\gamma c^{m}} ; m \geq 1$

Where $\beta_{0}$ is the attraction per $c=0$ and $\gamma$ is the illuminated saturation immersion coefficient, and $\gamma=[0, \infty]$, by and large $\gamma=1$, some circumstance $\beta_{0}$ is taken as $\beta_{0}=1$, and $m=2$ by reason of the most fireflies are visible just to a constrained separation. Consume arrangement room to be n-dimensional, the distance between any two fireflies $i$ and $j$ at $x_{i}$ and $x_{j}$, here for separation count, utilized Cartesian separation as, for separation count, utilized Cartesian separation as,

$c=\sqrt{\sum_{k=1}^{d}\left(x_{i}-x_{j}\right)^{2}}$

Here, time varying adaptive weight is developed for updating the position of fireflies. The AW linearly decreases with respect to time. Following equation is used for updating AW;

$\mathrm{S}_{\text {new }}(\mathrm{t}+1)=$

$\mathrm{w}(\mathrm{t}) \mathrm{S}_{\text {old }}+\beta_{0} \mathrm{e}^{-\gamma \mathrm{c}^{\mathrm{m}}}\left(\mathrm{x}_{\mathrm{j}}-\mathrm{x}_{\mathrm{i}}\right)+\alpha \varepsilon_{\mathrm{i}}$

Where $S_{\text {new }}(t+1)$ stands the position of the $i^{t h}$ firefly, after $t+1^{\text {th }}$ movement; $\alpha$ being the randomization parameter, $\alpha \in[0,1]$ for most problems, we can use a fixed value of $\alpha=0.1$. Here $\varepsilon_{i}$ is a vector of random numbers, which is come from Gaussian distribution. 


\subsection{Adaptive Weight (Inertia Weight Linearly Decreasing)}

Most of the firefly variants use time-varying adaptive weight (AW) strategies in which adaptive weight value are determined based on iteration number. Time-varying inertia weight approaches have important applications in numerous fields these procedures can be either increasing or decreasing and linear or non-linear. In, a linear decreasing $\mathrm{AW}$ is introduced to improve the fine tuning firefly's characteristics.

Many works have been done by researchers to increase its efficiency while handling optimization problems. Such work is introduced in original firefly characteristics to control its misuse and analysis for better performance. Still, adaptive weight firefly algorithm is known to have premature limitation convergence by solving difficult (multipeak) problems due to the lack of adequate energy. The addressing challenge has been incurred in a long time and has concerned much researcher's attention in global optimization field.

In principle that a significant inertia weight simplifies a global search although a small inertia weight simplifies a local search, a linearly reducing inertia weight was used with an initial value 0.9 and final value 0.4. By these values, inertia weight can be inferred as medium flexibility in which a firefly moves, showing that setting it to a comparatively high initial value (e.g., 0.9) makes fireflies performs efficiently and move in a low viscosity medium. Progressively decreasing it to a much lower value e.g., 0.4 makes firefly execute more exploitation and moves in a high viscosity medium.

In this method, $\omega$ the value is linearly decreased from an initial value $\left(W_{\max }\right)$ to final value $\left(W_{\min }\right)$ according to following equation:

$w(t)=\frac{w_{\text {max }}\left(w_{\text {max }}-w_{\min }\right) * t}{\operatorname{Max} G}$

Where $w(t) \in[0,1]$ is the inertia weight at $t$ iteration, $w_{\max }$ is the initial values of inertia weight, $w_{\min }$ is the final value of inertia weight, $t$ is current iteration and $\operatorname{Max} G$ is the maximum number of allowable iterations.

Thus the update of the $i^{\text {th }}$ firefly is formulated as follows,

$$
S_{\text {new }}=S_{\text {old }}+S_{\text {new }}(t+1)
$$

The equation (4) and (5) shows that the $i^{\text {th }}$ firefly, will move towards the $j^{\text {th }}$ firefly and which is more attractive one in the optimization. Thus the optimization using IWF reduces the complexity by achieving better performance. The experimental results demonstrate that the firefly joined quickly towards optimal positions but slowed down it is convergence speed when it is near optima. Thus, by using linearly decreasing inertia weight, firefly lacks global search capability at the end of run even in some cases when global search capability is essential to local minimum jump out. Accordingly, using adapting scheme for altering inertia weight was recommended to increase firefly's routine local optima.

The flowchart of IWF coupling with CFD is shown in Fig. 2. It can be seen that the MATLAB is the UDP server, and the FLUENT is the UDP client. The CFD program will be continually invoked automatically until the IWF program converges, the IWF program will be suspended when the CFD program is running. The shared files are used to exchange data between the IWF and CFD.

\section{RESULTS AND DISCUSSION}

In the present work, a new optimization method combining IWF and CFD is proposed to optimize the porosity of porous insert filled in an enhanced tube for thermo-hydraulic performance enhancement. The optimization results are discussed in detail as below.

The thermo-hydraulic performances of the enhanced tube by using porous inserts with optimized porosity ( $\varepsilon_{\text {opt }}$ ) at $\mathrm{Re}=800$ are shown in Table 1. For comparison purposes, the performances of the enhanced tube with some nonoptimized porous inserts are also listed in Table 1. It can be seen that the PEC and $\mathrm{Nu}$ of the enhanced tube can be greatly improved by using the optimized porous inserts, and the $\mathrm{f}$ can also be effectively reduced.

It also can be seen that the optimum PEC and the corresponding $\mathrm{Nu}$ both increase with the increase of $\mathrm{N}$. But the increase is slow when $\mathrm{N}>5$. The optimum PEC and the corresponding $\mathrm{Nu}$ by using multiple layers of optimized porous insert are both about 2.5 times higher than by using one layer of optimized porous insert.

Fig. 3 shows the distributions of optimum porosity and the corresponding dimensionless axial velocity $\left(\mathrm{U}=\mathrm{u} / \mathrm{u}_{\max }\right)$ at the cross section of the enhanced tube with different layers of optimized porous insert. It can be seen that the optimum porosity varies from 0.95 to 1.0 . The optimized multiple layers of porous insert can improve the thermal hydraulic performance even further.

It also can be seen from Fig. 3 that the velocity field can be rebuilt by inserting multiple layers of the porous media with different porosities, and the optimum porosity significantly increases the velocity and the velocity gradient near the wall with the increase of $\mathrm{N}$. Therefore, there are two main causes for the thermal hydraulic performance enhancement by inserting multiple layers of porous media:

(1) the multiple layers of porous media can increase the effective thermal conductivity;

(2) the multiple layers of porous media can also increase the velocity and the velocity gradient near the wall. 
However, the optimized axial velocity distribution almost does not change with the layer number of porous insert when $\mathrm{N}>5$. That is to say, there is an appropriate layer number of porous insert to ensure the optimal performance of the enhanced tube for a given set of parameters.

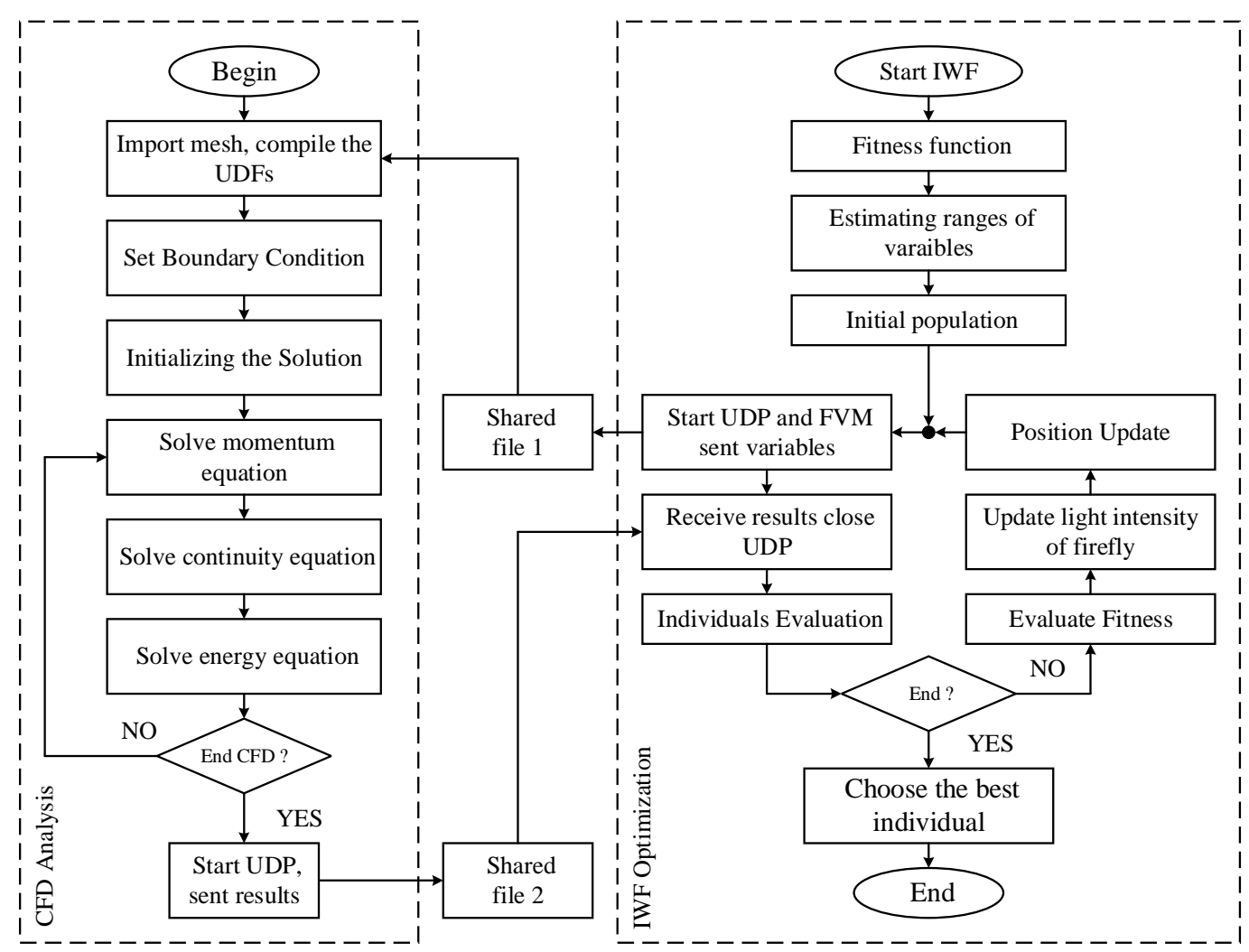

Fig. 2. Flowchart of $\bar{I} \bar{W} \bar{F}$ coupling with $\bar{C} \overline{F D}$.

Table 1 Performances of the enhanced tube with optimized and non-optimized porous inserts

\begin{tabular}{|c|c|c|c|c|c|c|}
\hline \multirow{2}{*}{ Cases } & $\mathrm{N}=1$ & $\mathrm{~N}=2$ & $\mathrm{~N}=3$ & $\mathrm{~N}=4$ & $\mathrm{~N}=5$ & $\mathrm{~N}=6$ \\
\cline { 2 - 7 } & $\varepsilon=.5$ & $\varepsilon=.9$ & $\varepsilon_{\text {opt }}$ & $\varepsilon_{\text {opt }}$ & $\varepsilon_{\text {opt }}$ & $\varepsilon_{\text {opt }}$ \\
\hline PEC & 0.47 & 2.58 & 9.98 & 21.4 & 24.6 & 26.0 \\
\hline $\mathrm{Nu}$ & 39.1 & 84.3 & 179 & 389 & 450 & 451 \\
\hline $\mathrm{f}$ & 523. & 32.2 & 5.35 & 5.54 & 5.61 & 5.60 \\
\hline
\end{tabular}

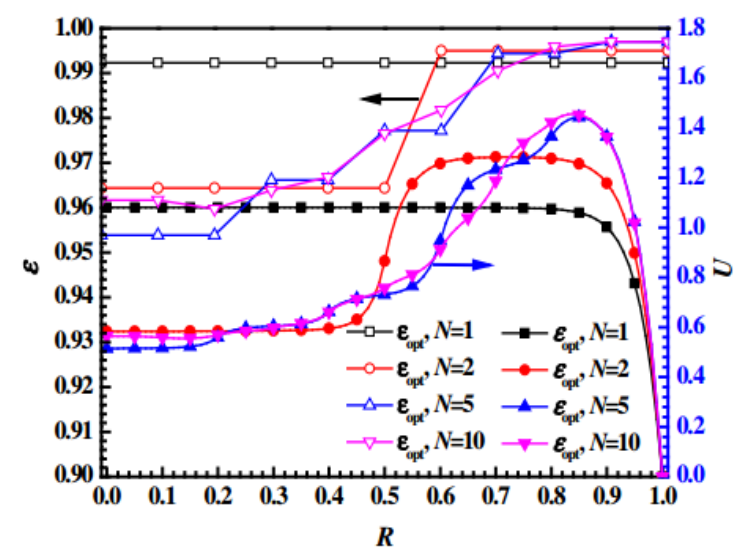

Fig. 3. Optimum porosity distributions and the corresponding axial velocity distributions at the cross section of the enhanced tube 


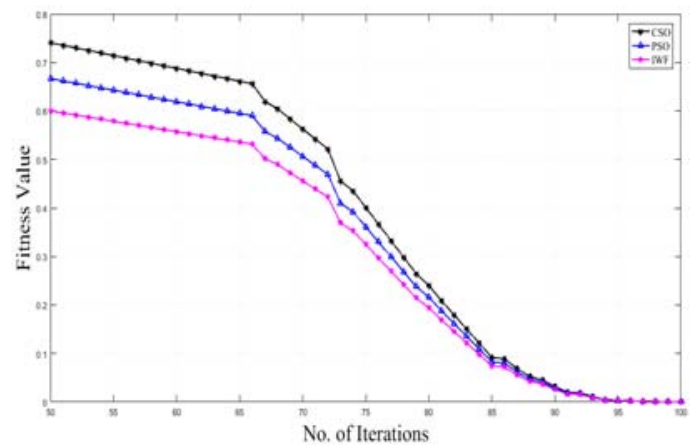

Fig. 4. Convergence Graph

\section{CONCLUSIONS}

In this paper, a new optimization method coupling IWF and CFD was found to optimize the configurations of porous insert in tubes to get a better thermo-hydraulic performance. The thermohydraulic performance of the enhanced tube can be increased effectively by using the optimized porous insert. The optimum porosity varies from 0.95 to 1.0. Using the optimized multiple layers of porous inserts can further increase the thermo-hydraulic performance, the optimum PEC of enhanced tube by using multiple layers of optimized porous insert is about 2.5 times higher than by using one layer of optimized porous insert at $\mathrm{Re}=800$.

\section{REFERENCES}

Bejan, A. (2004). Designed porous media: maximal heat transfer density at decreasing length scales, Journal of Heat and Mass Transfer 47(14), 3073-3083.

Bhargavi, D. and V. V. Satyamurty (2011). Optimum porous insert configurations for enhanced heat transfer in channels, Journal of Porous Media 14(3), 187-203.

Cheng, Z. D., Y. L. He and F. Q. Cui (2012). Numerical study of heat transfer enhancement by unilateral longitudinal vortex generators inside parabolic trough solar receivers, International journal of heat and mass transfer 55(21-22), 5631-5641.

He, Y. L., J. Xiao, Z. D. Cheng and Y. B. Tao (2011). A MCRT and FVM coupled simulation method for energy conversion process in parabolic trough solar collector, Renewable Energy 36(3), 976-985.

Maerefat, M., S. Y. Mahmoudi, and K. Mazaheri (2010). Numerical simulation of forced convection enhancement in a pipe by porous inserts. Heat Transfer Engineering 32(1), 4556.

Mahmoudi, Y. and N. Karimi (2014). Numerical investigation of heat transfer enhancement in a pipe partially filled with a porous material under local thermal non-equilibrium condition,
International Journal of Heat and Mass Transfer 68(1), 161-173.

Sathish, T. (2017a). Performance Measurement on Extracted Bio-Diesel from Waste Plastic. Journal of Applied Fluid Mechanics 10, 41-50.

Sathish, T. (2017b). Heat Transfer Analysis of Nano-fluid Flow In A Converging Nozzle With Different Aspect Ratios. Journal of New Materials for Electrochemical Systems 20, 161-167.

Sathish, T., and A. Muthulakshmanan (2018). Design and Simulation of Connecting Rods with Several Test Cases Using Al Alloys and High Tensile Steel. International Journal of Mechanical and Production Engineering Research and Development 8(1), 1119-1126.

Sathish, T. and J. Jayaprakash (2015). MetaHeuristic Approach to Solve Multi Period Disassembly-To-Order Problem of End-OfLife Products using Adaptive Genetic Algorithm. International Journal of Mechanical \& Mechatronics Engineering 15(3), 59-67.

Sathish, T., and J. Jayaprakash (2017a). Multi period disassembly-to-order of end-of-life product based on scheduling to maximise the profit in reverse logistic operation. International Journal of Logistics Systems and Management 26(3), 402-419.

Sathish, T., and J. Jayaprakash (2017b). Optimizing Supply Chain in Reverse Logistics. International Journal of Mechanical and Production Engineering Research and Development 7(6), 551-560.

Sivakumar, V., K. Visagavel and A. Selvakumar (2017). Analysis of Ventilation Rate in Cross Ventilated Rooms by Varying Aperture Shape of Windows using CFD. Journal of Applied Fluid Mechanics 10, 61-68.

Tao, W. Q. (2001). Numerical Heat Transfer, Xi'an Jiaotong University Press, Xi’an.

Tao, W. Q., Y. L He, Q. W. Wang, Z. G. Qu and F. Q. Song (2002). A unified analysis on 
T. Sathish and V. Mohanavel /JAFM, Vol. 11, Special Issue, pp. 31-37, 2018.

enhancing single phase convective heat transfer with field synergy principle, International Journal of Heat and Mass Transfer 45(24), 4871-4879.

Vafai, K. (2009). Handbook of Porous Media, CRC Press, New York.

Xi, H., M. J. Li, C. Xu, and Y. L. He (2013). Parametric optimization of regenerative organic Rankine cycle (ORC) for low grade waste heat recovery using genetic algorithm. Energy 58(1), 473-482.
Yang, C., A. Nakayama, and W. Liu (2011). Heat transfer performance assessment for forced convection in a tube partially filled with a porous medium, International Journal of Thermal Sciences 54(1), 98-108.

Yang, S. M., and W. Q. Tao, (2006). Heat Transfer, Higher Education Press, Beijing.

Zheng, Z. Z., Y. L. He, and Y. S. Li (2014). An entransy dissipation-based optimization principle for solar power tower plants. Science China Technological Sciences 57(4), 773-783. 